\title{
Use of cutaneous silent period parameters as a diagnostic indicator in patients with restless legs syndrome-a study in south Indian population
}

\author{
Alexander $\mathbf{R}^{1}$., Saraswathy $\mathbf{L}^{2}$, Gopinath $\mathrm{S.}^{3}$ \\ ${ }^{1}$ Dr Reena Alexander, Past Resident, Department of Physiology, Amrita institute of Medical sciences, Kochi, India. \\ Presently Assistant Professor, Department of Physiology, Sree Narayana Institute of Medical Sciences, Kochi, India, ${ }^{2}$ Dr \\ Saraswathy L, Professor \& Head, Department of Physiology, Amrita Institute of Medical Sciences, Kochi, India, ${ }^{3}$ Dr \\ Siby Gopinath, Professor, Department of Neurology, Amrita institute of Medical sciences, Kochi, Kerala, India.
}

Corresponding Author- Dr Reena Alexander, MBBS, MD, Past resident, Department of Physiology, Amrita institute of Medical Sciences, Kochi, Kerala, India. Email: drreenaalexander@gmail.com

\begin{abstract}
Objective: The objectives of this study were to investigate cutaneous-silent- period (CSP) parameters- CSP latency and CSP duration in patients with 'Restless legs Syndrome' and compare it with that of normal healthy controls. Methods: Thirty patients diagnosed with restless legs syndrome were included in this study. The cutaneous silent period was evoked by electrical stimulation of sural nerve whereby both CSP parameters CSP latency and CSP duration were recorded and analyzed. Results: The mean latencies of CSP among the thirty RLS patients were 101.95 \pm 12.12 while the control group showed $96.23 \pm 9.16$. There is a statistically significant high average latency in RLS patients (101.95 \pm 12.12) than control group (96.23 \pm 9.16$)$ with $\mathrm{p}$ value $=0.044$. This study also showed the average CSP duration to be high in RLS patients (42.25 \pm 11.12 ), when compared to the control group $-36.75 \pm 8.35$ ( $\mathrm{p}$ value=0.035). Conclusion: While RLS is a common sensorimotor disorder, the diagnosis of RLS is purely clinical and there are no objective tests for the diagnosis. Unfortunately, the awareness is poor among the lay population, general practitioners and even specialists in the Indian subcontinent. The authors feel that the CSP test can be used as an objective method supporting the diagnosis of RLS. Furthermore, we believe that this paper will help create awareness in addition to encouraging more research in this arena from medical personnel in India.
\end{abstract}

Key words- Cutaneous silent period, Restless leg Syndrome, Small fiber neuropathy, Willis-Ekbom disease.

\section{Introduction}

The first known medical description of 'Restless legs Syndrome' commonly referred to as RLS was by the discoverer of the famous 'circle of Willis', Sir Thomas Willis [1]. Almost three centuries after Willis in 1945, Karl-Axel Ekbom coined the term 'Restless legs syndrome' and provided a detailed and comprehensive report of this condition in his doctoral thesis [2]. Prior to 1990, RLS was a grossly under-diagnosed sensorimotor disorder with prevalence rate of $1-5 \%$ [3]. However, following the description of diagnostic criterion by the International RLS Study Group in 1995, higher prevalence rates have been reported [4]. While studies on Indian population on RLS are limited and the general prevalence of RLS is unknown, two papers have

Manuscript received: $07^{\text {th }}$ February 2018

Reviewed: $12^{\text {th }}$ February 2018

Author Corrected: $16^{\text {th }}$ February 2018

Accepted for Publication: $21^{\text {st }}$ February 2018 reported rates of $2.1 \%$ and $6.25 \%$ in different patient groups, possibly from poor awareness and lack of objective criteria $[5,6]$. The need for an objective test to better the diagnose RLS led to the cutaneous silent period (CSP) first described by W.W. Hoffmann in 1922, being used as a noninvasive test for RLS patients [7]. This study focuses on the use of CSP as a diagnostic indicator of RLS in thirty patients and raises the hope of higher awareness and better diagnosis and treatment for patients with RLS who often remain undiagnosed in the general population.

\section{Materials and Methods}

Place of study- This study was conducted in Amrita institute of medical sciences, Kochi from September 2012 to January 2014. 


\section{Original Research Article}

Type of study- This was a prospective case control study

Sample collection- Thirty subjects who were diagnosed with RLS were included in this study. Thirty normal healthy volunteers who visited our general health checkup center and were subsequently confirmed to be free of any medical problems were recruited and used as control subjects.

Sampling methods- All patients with RLS were checked through work-up for evidence of the presence of a disease generally considered to be associated with the symptoms of RLS or peripheral neuropathy, such as uremia, iron deficiency, diabetes, and rheumatoid arthritis. These patients were also subjected to detailed history taking, questionnaire screening for RLS, clinical examination, laboratory examination and review of old medical records. Cutaneous silent period was recorded by the electromyographer. This study was approved by the ethics review committee and consent was obtained from all participants.

Inclusion criteria- These patients fulfilled the four essential criteria [3],[4] for RLS diagnosis -1) an urge to move the legs, usually accompanied by uncomfortable sensations, 2) the urge to move or unpleasant sensations begin or worsen during periods of rest or inactivity, 3) the urge to move or unpleasant sensations are partially or completely relieved by movement, and 4) worsening of symptoms in the evening or night, as described by the International RLS study group.

Exclusion criteria- Patients with muscular diseases, neuromuscular junction disorder, malignancy, rheumatoid arthritis, osteoarthritis, leg cramps, long term drug use, vascular disease, such as deep venous thrombosis were excluded from the study.

Measurement of CSP for Lower limb- The parameters measured in studies of the cutaneous silent period were the CSP latency and CSP duration. Recordings were performed using surface electrodes attached in a belly-tendon fashion. In studies on the sural nerve at the lower limbs, the tibialis anterior muscle was used for recording using a Nicolet Viking select II EMG instrument: active electrode was placed over the tibialis anterior muscle and reference over the tendon of tibialis anterior. The equipment settings were as follows- Time base: $50 \mathrm{~ms}$, Sensitivity: $1-2 \mathrm{mV}$, Filter: $2 \mathrm{~Hz}-10 \mathrm{KHz}$, Duration: $2 \mathrm{~ms}$, Rate: $1 \mathrm{HZ}$, Notch filter: $50 \mathrm{~Hz}$. The stimulation site was one $\mathrm{cm}$ below and behind the lateral malleolus over the sural nerve. Stimulation was carried out with a standard painful stimulus through a bar electrode fixed behind the lateral malleolus and the response was recorded with an electrode fixed on the belly of the contracting tibialis anterior muscle.

Ground electrode was placed in between active electrode and cathode. During maximal voluntary contraction single stimuli at painful intensities of $0.5 \mathrm{~ms}$ duration were delivered behind the lateral malleolus over the sural nerve until a complete silent period of reproducible latency and duration was obtained. To obtain steady maximal contraction, the subject was asked to contract against resistance, and an EMG audio signal was used to monitor the muscle contraction. The CSP was repeatedly measured. Every waveform was carefully inspected, and five recordings showing complete silencing of motor unit potential, with longest duration and shortest latency were selected. The beginning and endpoint latencies of the CSP were identified by visual inspection at the beginning of an abrupt decrease or upon the return of EMG activity, respectively.

CSP duration was defined as the time between the beginning and endpoint latencies. The average latencies and durations (the average values of the five recordings) were used in the final analysis. The motor nerve conduction study was performed by stimulating the peroneal and posterior tibial nerves at the ankle and knee, with recordings at the extensor digitorum brevis and abductor hallucis muscle, respectively. The sensory nerve conduction study was performed with sural nerve stimulation and recording electrode was placed posterior to lateral malleolus.

Statistical analysis- Statistical analysis was done using IBM SPSS statistics 20 windows. For all continuous variables the results are given in mean \pm SD and for categorical variables as percentage. To compare average of continuous variables those following normal distribution, independent sample t- test was performed. For finding association between two categorical variables Chi square test was used. P-values of $<0.05$ were considered significant.

\section{Results}

We studied 60 subjects which included thirty patients with RLS who were compared with thirty normal subjects. The diagnosis of RLS was made on the four essential criteria for RLS as described by the International RLS study group. RLS 


\section{Original Research Article}

was diagnosed if the patients answered affirmatively to all questions eliciting answers to the four essential National Institutes of Health/International Restless Legs Syndrome Study Group (NIH/ IRLSSG) criteria for diagnosis of RLS. These patients were asked questions regarding other features of RLS including sleep disturbance in the form of delayed onset of sleep, and leg movements in sleep as described by their spouse. They were also administered the IRLSSG severity scoring scale to assess the severity of RLS. Patients were investigated to determine any secondary cause of RLS. Out of the 30 patients 5 were diabetic, 4 had radiculopathy, 2 had iron deficiency anemia, 2 had hyperuricemia and 1 patient suffered from renal failure. Nerve conduction studies in 26 patients were normal.

Mean age for RLS patients was $50.23 \pm 15.19$ years; mean age for control subject group was $44.30 \pm 12.82$ years. The age group range of RLS patients were 23-70 years and control group were 23 - 68 years. While in the RLS group $43.3 \%$ were females and $56.7 \%$ were males, among the control group $43 \%$ were females and $56.7 \%$ were males $(p=1.000)$. There was no significant difference in gender or age between two groups. Out of the 30 patients diagnosed with RLS, according to the severity, $7 \%$ had mild form of RLS, $33 \%$ had moderate, $40 \%$ had severe and $20 \%$ had very severe affection on the IRLS severity scale (Graph 1). The mean latencies of CSP among RLS patients $101.95 \pm 12.12$ and the control group was $96.23 \pm 9.16$. There was a statistically significant high average latency in RLS patients (101.95 \pm 12.12$)$ than control group $(96.23 \pm 9.16) \mathrm{p}$ value $=0.044$ (Table 1) (Graph 2). Average duration was high in RLS patients- 42.25 \pm 11.12 , then the control group $-36.75 \pm 8.35(\mathrm{p}$ value $=0.035)($ Table 2) $($ Graph 3$)$

Table-1: Cutaneous nerve silent period latency for RLS patients and controls

\begin{tabular}{|c|c|}
\hline RLS & $101.95 \pm 12.1$ \\
\hline CONTROL & $96.23 \pm 9.16$ \\
\hline
\end{tabular}

$\mathrm{p}$ value $=0.044$

Table-2: Cutaneous nerve silent period duration for RLS patients and controls

\begin{tabular}{|c|c|}
\hline RLS & $42.25 \pm 11.12$ \\
\hline CONTROL & $36.75 \pm 8.35$ \\
\hline
\end{tabular}

$\mathrm{p}$ value $=0.035$

Graph-1: IRLS severity rating scale

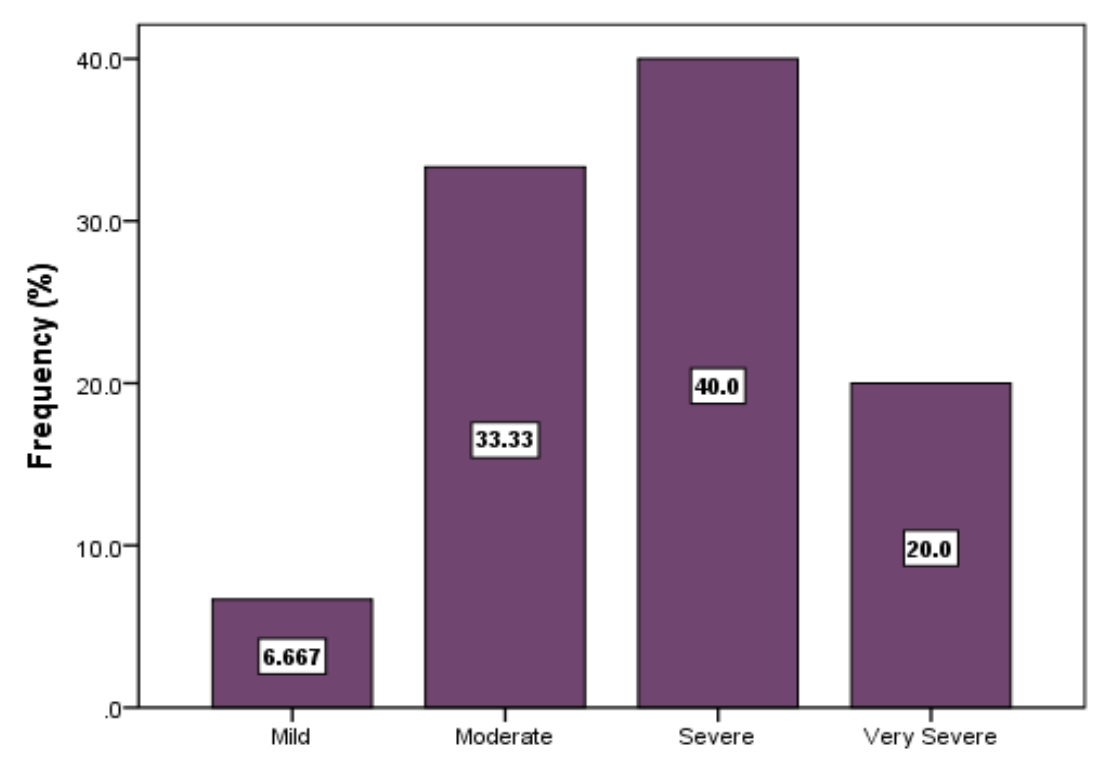

IRLS

Graph-2: Cutaneous nerve silent period latency for RLS patients and controls 


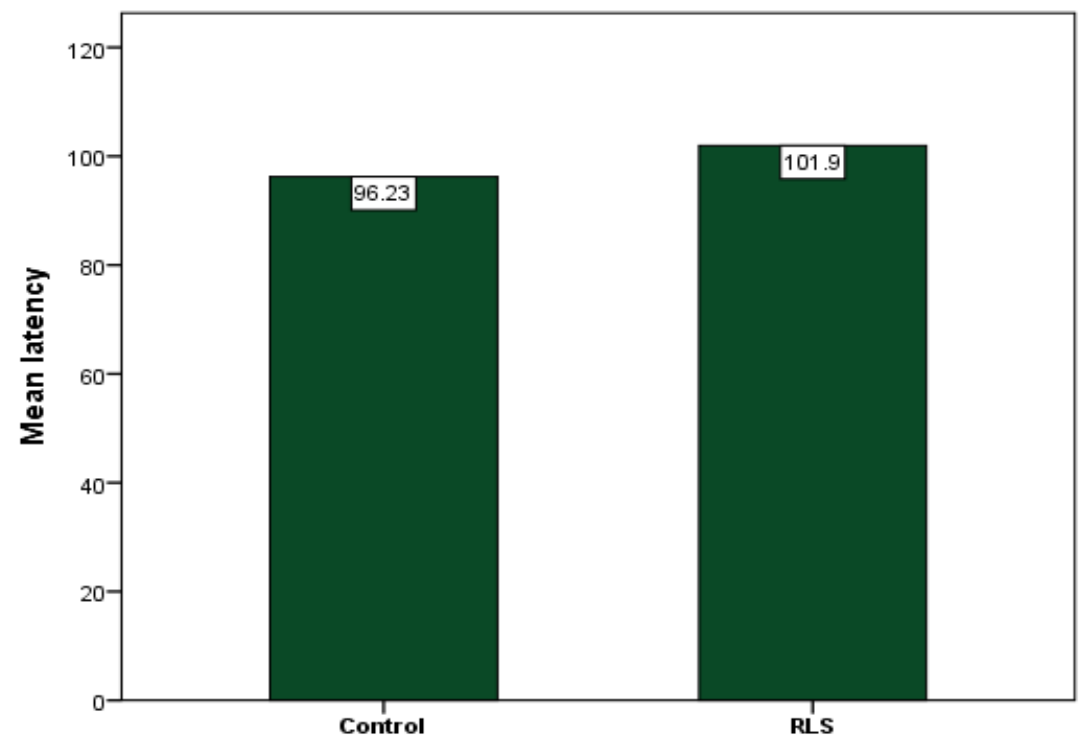

Graph-3: Cutaneous nerve silent period duration for RLS patients and controls

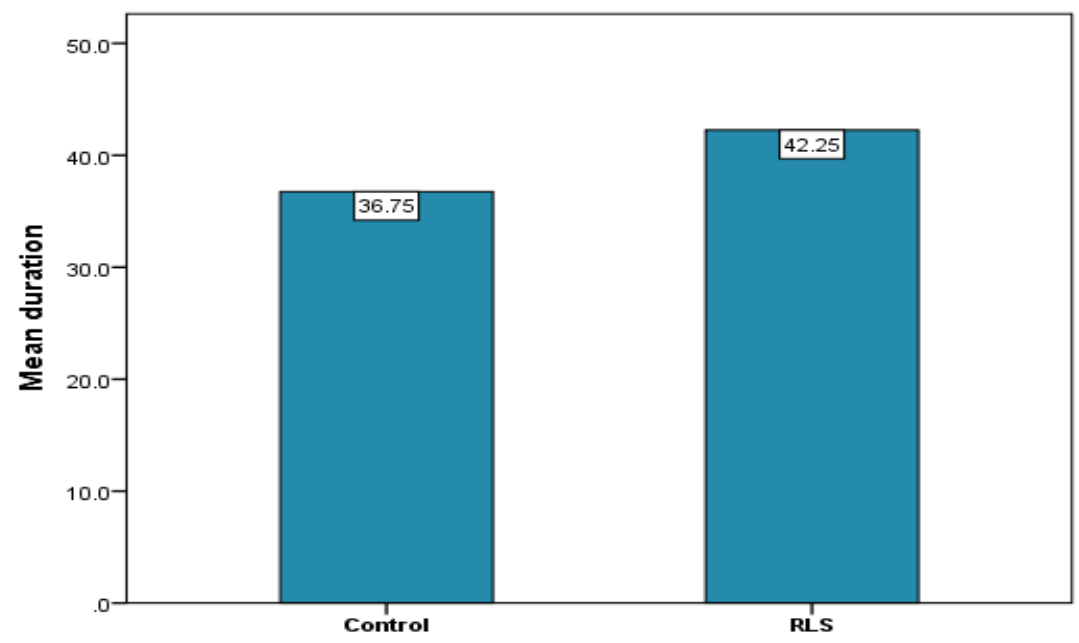

\section{Discussion}

Restless legs Syndrome is a sensorimotor disorder characterized by an intense irresistible urge to move the legs and is accompanied by unpleasant paresthetic sensations which begin or worsen with rest, but gets relieved partially or completely with movement [8]. Though seemingly benign, the unfortunate patient with RLS ends up with sleep disturbance, fatigue and inability to tolerate a sedentary life that ultimately impacts his social, occupational and family life leading to severe stress, frustration and unhappiness in life. Gupta R et, al, in an epidemiological study paper on 653 patients with insomnia or leg pain, shocked the medical community by titling the paper as 'An Indian experience of RLS' and revealing that though RLS was a common disorder in India, it was rarely diagnosed and barely treated even by specialists like neurologists and psychiatrists and sometimes treated by medicines that were either ineffective or even deleterious to RLS patients [9]. It is therefore imperative that RLS which is a common under-diagnosed condition in India be recognized at an early stage of its inception and prompt treatment be started to improve the patient's 'quality of life'. The diagnosis of RLS primarily depends on the clinical criteria and the only instrumental tool, the suggested immobilization test leads to equivocal results [10]. Some authors have suggested the use of F- wave duration and its ratio with the corresponding muscle action potential as a possible supportive diagnostic tool for RLS patients: both of these were significantly longer when compared to controls $[10,11]$.

Cutaneous silent period or CSP is a brief pause in motor unit potentials following painful cutaneous stimuli that propagate through high threshold afferents that might inhibit anterior horn cells via polymodal inter-neuronal 


\section{Original Research Article}

synapses. CSP measurement is a simple non invasive method that studies the small peripheral nerve fibers (Adelta and $\mathrm{C}$ ), and this could be an objective test that may help better diagnose RLS in patients [12]. A review of literature reveals that cutaneous silent period parameters have been studied in a variety of neuropathological conditions viz: Friedreich's ataxia, entrapment syndromes, neuropathies, Parkinson's disease, dystonias and pyramidal syndromes besides others $[13,14]$. Studies of CSP in RLS are limited in the literature with conflicting results. $\mathrm{Oz} \mathrm{O}$ et al, [12] found that while the CSP latency was longer, the CSP duration was shorter in RLS patients compared to controls. While similar findings were reported by Isak B et al, [11] a recent study by Congui $\mathrm{P}$ et al, [10] did not find any significant abnormality in CSP in RLS patients probably due to the small sample size or minor differences in the methodology. Han JK et al, [15] investigated the patho-physiological relationship between RLS and small fiber neuropathy using the CSP and found that while the CSP latencies did not differ between RLS patients and healthy controls, the mean CSP duration was significantly longer in RLS patients than in controls. In our study both latency and duration of CSP in RLS patients were high compared to that of the controls. The mean latencies among RLS patients $101.95 \pm 12.12$ and the control group were 96.23 \pm 9.16 ( $\mathrm{p}$ value $=0.044$ ). Average CSP duration was high in RLS patients- $42.25 \pm 11.12$, then the control group $36.75 \pm 8.35(\mathrm{p}$ value $=0.035)$. Polydefkis $\mathrm{M}$ et al, suggested that the prolonged CSP latency may be the result of a loss of function in the spinal inhibitory circuit associated with small fibre neuropathy and reduction in the conduction velocities of afferent A- delta fibres [16]. Gemignani et al, [17] reported a high incidence of RLS in patients with polyneuropathy than in patients without and most RLS patients were clinically compatible with small fiber neuropathy. These authors and others [15,18,19.20] suggested that the pathomechanisms of RLS are activated by peripherally disrupted sensory modulation as well as by the hypofunctioning of the dopaminergic hypothalamo-spinal pathways that exert modulatory actions on the polymodal interneuronal synapses: the pause time of the motor unit potential namely CSP duration, will be prolonged if the descending fibers does not appropriately modulate the inhibition of the motor unit potential in response to the painful stimuli applied. Han $\mathrm{J} \mathrm{k}$ et al, [15] also found that the prolonged CSP duration significantly improved to the levels of the control group along with a decrease in the mean TLS rating score after dopamine agonist medication, thereby implying that the CSP duration changes could be useful as a clinical measure of improvement following dopamine agonist therapy. The dopamine supply is believed to compensate for the hypo-functioning of the dopaminergic hypothalamic pathway resulting in improvement of the prolonged CSP duration. The present study of CSP in RLS patients may possibly be the first from the south Indian population and could pilot the way for more detailed neuro-physiological studies in RLS patients.

\section{Conclusion}

RLS patients in India have been particularly unfortunate as it is a seriously under-diagnosed condition. Early detection will save the patient from intense psychological and emotional trauma and therefore there is a need to devise newer investigations in this direction. CSP is a relatively simple electro-diagnostic test which though needing a painful stimulus is often well tolerated. The abnormal CSP parameter can help objectively identify patients with clinical diagnosis of RLS therapy leading to an early treatment option. While this was a hospital-based study with a small sample size, the effects of medication on the CSP parameters were not investigated. We feel that there is a need to further investigate CSP in RLS patients by increasing the sample size and comparing the cutaneous silent period parameters before and after treatment. While the first, second and third authors investigated the clinical aspects of each patient, the third author selected and did an exhaustive electromyography evaluation and correlated it clinically with the patient groups. The first author was also involved in exhaustive literature search and writing up the paper.

Study outcome- We believe that this study will encourage others to further investigate the use of CSP in RLS patients with an aim to corroborate these findings. This study will also help to create better awareness and management of RLS patients in the Indian subcontinent.

Acknowledgment- Thanks to the technicians of the department of neurophysiology, Amrita hospital. Presently the first author is working in department of Physiology, Sree Narayana institute of Medical sciences, Kochi.

Funding: Nil, Conflict of interest: None. Permission of IRB: Yes

\section{References}

1. Coccagna G, Vetrugno R, Lombardi C, Provini F. Restless legs syndrome: an historical note. Sleep Med. 2004 May;5(3):279-83. 


\section{Original Research Article}

2. Ekbom KA: Restless legs: a clinical study. Acta Med Scand 1945; 158(suppl):1-122.

3. Walters AS. Toward a better definition of the restless legs syndrome. The International Restless Legs Syndrome Study Group. Mov Disord. 1995 Sep;10(5):634-42.

4. Lavigne GJ, Montplaisir JY. Restless legs syndrome and sleep bruxism: prevalence and association among Canadians. Sleep. 1994 Dec;17(8):739-43.

5. Rangarajan S, Rangarajan S, D'Souza GA. Restless legs syndrome in an Indian urban population. Sleep Med. 2007 Dec;9(1):88-93. Epub 2007 Sep 6.

6. Rangarajan S, D'Souza GA. Restless legs syndrome in Indian patients having iron deficiency anemia in a tertiary care hospital. Sleep Med. 2007 Apr;8(3):24751. Epub 2007 Mar 26.

7. Angel RW, Hofmann WW, Eppler W. Silent period in patients with parkinsonian rigidity. Neurology. 1966 May;16(5):529-32.

8. Trenkwalder C, Paulus W, Walters AS. The restless legs syndrome. Lancet Neurol. 2005 Aug;4(8):465-75.

9. Gupta R, Lahan V, Goel D. Restless Legs Syndrome: a common disorder, but rarely diagnosed and barely treated--an Indian experience. Sleep Med. 2012 Aug;13(7):838-41. doi: 10.1016/j.sleep.2012.03.018. Epub 2012 Jun 14.

10. Congiu P, Fantini ML, Milioli G, Tacconi P, Figorilli M, Gioi G, Pereira B, Marrosu F, Parrino L, Puligheddu M. F-Wave Duration as a Specific and Sensitive Tool for the Diagnosis of Restless Legs Syndrome/Willis-Ekbom Disease. J Clin Sleep Med 2017 Mar 15; 13(3):369-75.

11. Isak B, Uluc K, Salcini C, Agan K, Tanridag T, Us O. A neurophysiological approach to the complex organisation of the spine: F-wave duration and the cutaneous silent period in restless legs syndrome. Clin Neurophysiol. 2011 Feb;122(2):383-90. doi: 10.1016/j.clinph.2010.07.005. Epub 2010 Aug 17.
12. Oz O, Erdoğan C, Yücel M, Akgün H, Kütükçü Y, Gökçil Z, Odabaşı Z. Effect of pramipexole on cutaneous-silent-period parameters in patients with restless legs syndrome. Clin Neurophysiol. 2012 Jan;123(1):154-9. doi: 10.1016/j.clinph.2011.05.027.

13. Tiric-Campara M, Denislic M, Djelilovic-Vranic J, et al. Cutaneous Silent Period in the Evaluation of Small Nerve Fibres. Med Arch. 2014;68(2):98-101. doi:10.5455/medarh.2014.68.98-101.

14. Uncini A, Kujirai T, Gluck B, Pullman S. Silent period induced by cutaneous stimulation. Electroencephalogr Clin Neurophysiol. 1991 Oct; 81(5):344-52.

15. Han JK, Oh K, Kim BJ, Koh SB, Kim JY, Park $\mathrm{KW}$, Lee DH. Cutaneous silent period in patients with restless leg syndrome. Clin Neurophysiol. 2007 Aug;118(8):1705-10. Epub 2007 Jun 22.

16. Polydefkis M, Allen RP, Hauer P, Earley CJ, Griffin JW, McArthur JC. Subclinical sensory neuropathy in late-onset restless legs syndrome. Neurology. 2000 Oct 24;55(8):1115-21.

17. Gemignani F, Brindani F, Negrotti A, Vitetta F, Alfieri S, Marbini A. Restless legs syndrome and polyneuropathy. Mov Disord. 2006 Aug;21(8):1254-7.

18. Gladwell SJ, Coote JH. Inhibitory and indirect excitatory effects of dopamine on sympathetic preganglionic neurons in the neonatal rat spinal cord in vitro. Brain Res. 1999 Feb 13;818(2):397-407

19. Clemens S, Rye D, Hochman S. Restless legs syndrome: revisiting the dopamine hypothesis from the spinal cord perspective. Neurology. 2006 Jul $11 ; 67(1): 125-30$.

20. Garraway SM, Hochman S. Modulatory actions of serotonin, norepinephrine, dopamine, and acetylcholine in spinal cord deep dorsal horn neurons. J Neurophysiol. 2001Nov;86(5):2183-94.

\section{How to cite this article?}

Alexander R., Saraswathy L, Gopinath S. Use of cutaneous silent period parameters as a diagnostic indicator in patients with restless legs syndrome-a study in south Indian population. Int J Med Res Rev 2018;6 (02):110-115. doi:10.17511/ijmrr.2018.i02.07. 